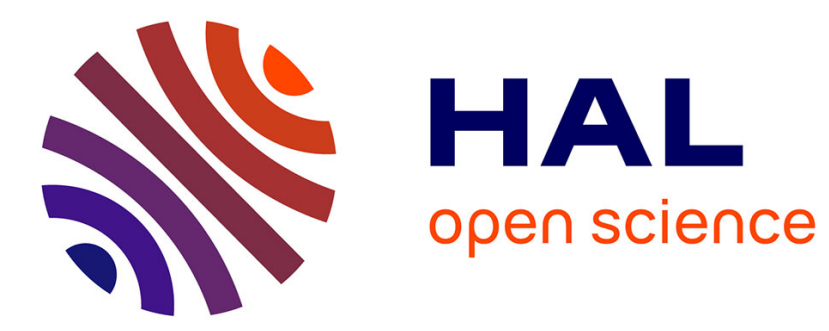

\title{
Progressive Current Source Models in Magnetic Vector Potential Finite Element Formulations
}

Patrick Dular, Patrick Kuo-Peng, Mauricio Valencia Ferreira da Luz, Laurent Krähenbühl

\section{- To cite this version:}

Patrick Dular, Patrick Kuo-Peng, Mauricio Valencia Ferreira da Luz, Laurent Krähenbühl. Progressive Current Source Models in Magnetic Vector Potential Finite Element Formulations. Compumag 2015, Jun 2015, Montréal, Canada. pp.648. hal-01174978

\section{HAL Id: hal-01174978 https://hal.science/hal-01174978}

Submitted on 31 Aug 2015

HAL is a multi-disciplinary open access archive for the deposit and dissemination of scientific research documents, whether they are published or not. The documents may come from teaching and research institutions in France or abroad, or from public or private research centers.
L'archive ouverte pluridisciplinaire HAL, est destinée au dépôt et à la diffusion de documents scientifiques de niveau recherche, publiés ou non, émanant des établissements d'enseignement et de recherche français ou étrangers, des laboratoires publics ou privés. 


\title{
Progressive Current Source Models in Magnetic Vector Potential Finite Element Formulations
}

\author{
Patrick Dular ${ }^{1,2}$, Patrick Kuo-Peng ${ }^{3}$, Mauricio V. Ferreira da Luz ${ }^{3}$, and Laurent Krähenbühl ${ }^{4}$ \\ ${ }^{1}$ University of Liege, ACE, Liège, Belgium, Patrick.Dular@ulg.ac.be $\quad{ }^{2}$ F.R.S.-FNRS, Belgium \\ ${ }^{3}$ Universidade Federal de Santa Catarina, GRUCAD, Brazil \\ ${ }^{4}$ Université de Lyon, Ampère (CNRS UMR5005), École Centrale de Lyon, Écully, France
}

\begin{abstract}
Progressive refinements of the current sources in magnetic vector potential finite element formulations are done with a subproblem method. The sources are first considered via magnetomotive force or Biot-Savart models up to their volume finite element models, from statics to dynamics. A novel way to define the source fields is proposed to lighten the computational efforts, via the conversion of the common volume sources to surface sources, with no need of any pre-resolution. Accuracy improvements are then efficiently obtained for local currents and fields, and global quantities, i.e. inductances, resistances, Joule losses and forces.
\end{abstract}

Index Terms-Finite element method, inductor, model refinement, subproblems.

\section{INTRODUCTION}

The current sources in finite element (FE) magnetodynamic problems can generally be considered via Biot-Savart (BS) models, possibly giving the conductors some simplified geometries, with, e.g., filament, circular or rectangular cross sections [1]. In the slots of magnetic core regions, an alternative to the explicit definition of such simplified geometries is to consider the slot-core interfaces as perfect magnetic walls via a zero flux boundary condition (BC). The slot regions are thus omitted from the calculation domain, neglecting the slot leakage flux instead of calculating other inaccurate distributions. The associated sources are magnetomotive forces (MMFs) [2].

MMF sources are inherently associated with surfaces whereas BS models define source fields (SFs) that are originally volume sources (VSs). A novel procedure is here proposed to convert the BS SFs into surface sources (SSs) as well, to lighten the computational efforts, mainly by reducing the number of BS evaluations. It is based on interface conditions (ICs) that define field discontinuities fixed from surface BS fields. The developments are performed in the frame of the magnetic vector potential $\boldsymbol{a}$ formulations. Accuracy improvements up to volume FE representations of the conductors, that improve the local field distributions, and from static to dynamic excitations, that accurately render skin and proximity effects, can be done at a second step via the subproblem (SP) method (SPM) [3], [4], which defines a general frame for the whole modeling procedure.

\section{Progressive Source Models - Methodology}

\section{A. MMF model}

Boundary $\Gamma_{m}$ of some magnetic (conducting or not) regions $\Omega_{m}$, possibly extended with air gaps $\Omega_{g} \subset \Omega_{m}$, can first be considered as a perfect magnetic wall (Fig. 1), thus with no leakage flux. For the so-defined SP $p \equiv \mathrm{MMF}$, the calculation domain is thus limited to $\Omega_{m}$, called a flux tube, with a BC on $\Gamma_{m}$ fixing a zero normal trace of the magnetic flux density $\boldsymbol{b}_{p}$. In terms of a magnetic vector potential $\boldsymbol{a}_{p}$, with $\boldsymbol{b}_{p}=\operatorname{curl} \boldsymbol{a}_{p}$, one has the equivalent essential BCs

$$
\left.\boldsymbol{n} \cdot \boldsymbol{b}_{p}\right|_{\Gamma_{m}}=0 \Leftrightarrow \boldsymbol{n} \times\left.\boldsymbol{a}_{p}\right|_{\Gamma_{m}}=\boldsymbol{n} \times\left.\operatorname{grad} w_{p}\right|_{\Gamma_{m}},
$$

where $\boldsymbol{n}$ is the exterior unit normal and $w_{p}$ is a multivalued unknown surface scalar potential. The required gauge condition on $\boldsymbol{a}$ allows to particularize the distribution of $w_{p}$.

Through such a process, the actual current source regions $\Omega_{s}$ are idealized as perfect solenoids winded all along $\Gamma_{m}$, i.e., around the flux tubes. In 3-D, scalar potential $w_{p}$ in $\mathrm{BC}(1 \mathrm{~b})$ can be reduced to a constant jump through each of the cut lines making $\Gamma_{m}$ simply connected. In 2-D, such constant jumps come down to the definition of a constant $\boldsymbol{a}_{p}$ (a kind of floating potential) on each non-connected portion $\Gamma_{m, i}$ (with $i$ the portion index) of $\Gamma_{m}$ (Fig. 1, left). The constant jumps are directly (strongly) related to the unknown magnetic fluxes flowing in $\Omega_{m}$ and are related to the MMFs via the weak formulation, tested with the non-local jump test functions [2]. An example of result is given in Fig. 3.

Each $\Gamma_{m, i}$ can be considered as the boundary of a slot in a device. The related MMF gathers all the current sources in the slot, for all coils, e.g., coils with different phases in a machine or primary/secondary coils in a transformer. A slot can be generalized to represent the exterior region, including coils as well.

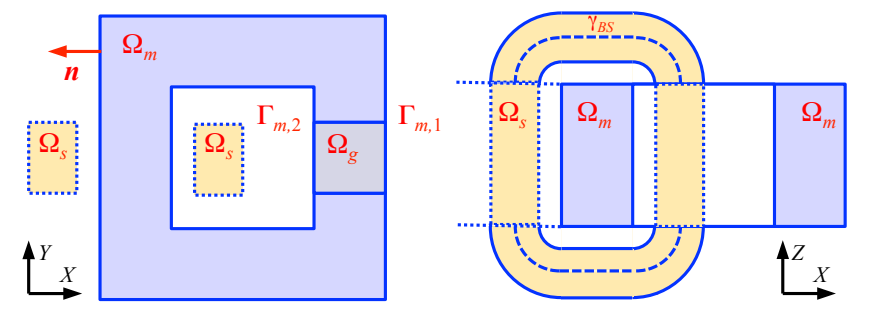

Fig. 1. Example of a magnetic region $\Omega_{m}$, including an air gap $\Omega_{g}$, first considered without leakage flux, via perfect magnetic walls BCs on $\Gamma_{m, 1}$ and $\Gamma_{m, 2}$ in channel slots (XY-plane, left) coupled to end windings coil $\gamma_{B S}$ via BS-SF model (XZ-plane, right).

\section{B. BS-SF model}

With the SPM, the BS SF evaluations can be limited to the material regions $\Omega_{m}$ via local VSs [3], [4], instead of the whole domain with the common method [1]. Such a support reduction already allows to lighten the BS calculations. Then, for accurate combinations with the reaction fields, the BS SFs gain at being projected onto similar function spaces (edge FEs for both source and reaction fields). Also, instead of volume 
projections of the SFs in the mesh of $\Omega_{m}$, the SFs gain at being calculated there via an FE problem with their boundary values as BCs on $\partial \Omega_{m}$, thus already limiting the BS evaluations to surfaces.

To go one step further, such a preliminary FE problem can be avoided through its inclusion in the main SP $p \equiv \mathrm{BS}-\mathrm{SF}$. The key is to think of two successive SPs $p$ a and $p$ b actually solved together (Fig. 2). SP $p$ a first prevents the field to enter $\Omega_{m}$, thus with a reaction field in $\Omega_{m}$ opposing the BS field (direct solution of SP $q$ ), keeping unchanged the field out of $\Omega_{m}$. This constraint is simply expressed via both tangential and normal field trace discontinuities of magnetic field $\boldsymbol{h}_{p \text { a }}$ and magnetic flux density $\boldsymbol{b}_{p \mathrm{a}}$ through $\Gamma_{m}$, i.e., with ICs with SSs

$$
\left[\boldsymbol{n} \times \boldsymbol{h}_{p \mathrm{a}}\right]_{\Gamma_{m}}=-\boldsymbol{n} \times\left.\boldsymbol{h}_{q}\right|_{\Gamma_{m}},\left[\boldsymbol{n} \cdot \boldsymbol{b}_{p \mathrm{a}}\right]_{\Gamma_{m}}=-\left.\boldsymbol{n} \cdot \boldsymbol{b}_{q}\right|_{\Gamma_{m}} .
$$

In terms of $\boldsymbol{a}_{p}$, IC (2b) leads to

$$
\left[\boldsymbol{n} \times \boldsymbol{a}_{p \mathrm{a}}\right]_{\Gamma_{m}}=-\boldsymbol{n} \times\left.\boldsymbol{a}_{q}\right|_{\Gamma_{m}} .
$$

The result is an exact zero field in $\Omega_{m}$, with no need of volume calculation. Then, SP $p$ b considers the actual physical properties in $\Omega_{m}$, with no more VSs, which is a great advantage. Combining SPs $p$ a and $p \mathrm{~b}$ thus gives a single SP $p$ that considers the physical properties of $\Omega_{m}$ and with the trace discontinuities of SP $p$ a (2a-b) still being valid for its solutions $\boldsymbol{h}_{p}$ and $\boldsymbol{b}_{p}$ (because SP $p \mathrm{~b}$ introduces no discontinuities).

BS SF evaluations are thus only required on $\Gamma_{m}$, which is a significant advantage. At the discrete level, the IC-SSs in (2ab)-(3) can be obtained from a mesh projection of only the $\boldsymbol{a}$ BS SF in a layer of FEs along the boundary of $\Omega_{m}$. Details will be given in the extended paper. An example of result is given in Fig 4.

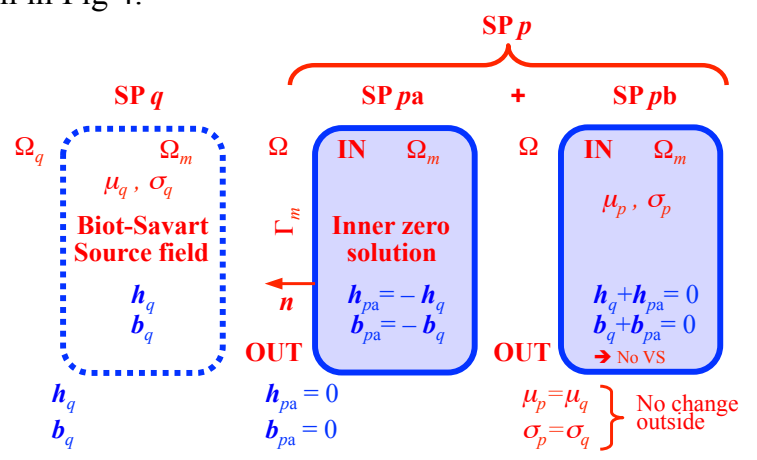

Fig. 2. BS SF (SP $q$ ) for a material region $\Omega_{m}$ (SP $p$ ): SP $p$ is split into SPs $p$ a and $p \mathrm{~b}$, simultaneously solved, SP $p$ a removing the volume BS solution $q$ inside $\Omega_{m}$ and SP $p$ b considering the actual properties of $\Omega_{m}$, with no need of VSs for change of properties, but with IC-SSs for the unified SP $p$.

\section{Coupling between MMF and BS-SF models}

In 3-D, the slots are generally of two types: 1) a channel surrounded by magnetic regions and possible air gaps (usually considered in 2-D models), coupled through interfaces to 2) an open exterior region for the end windings (3-D effects). It is here proposed to define the channel slots via the MMF model and the end winding regions via the actual consideration of the winding, e.g., via a BS model (Fig. 1, right). With such a coupling between MMF and BS-SF models, the flux wall surface has to be extended to these interfaces. Practical details will be given in the extended paper.

\section{Volume FE models of conductors}

Additional SPs for volume FE models of the source conductors can follow (Fig. 5) [3], [4], for accurate determination of their characteristics (impedances, losses, forces).

The proposed methodology has first been validated on 2-D test problems. It offers tools that allow to reduce the computational effort of the classical approach. When applied up to 3-D problems, its advantages will be shown to be numerous and significant, with quantification of the benefits.

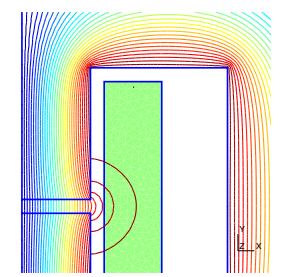

(a) total solution

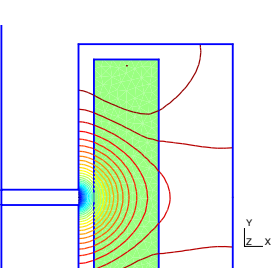

(b) total sol., in-slot zoom

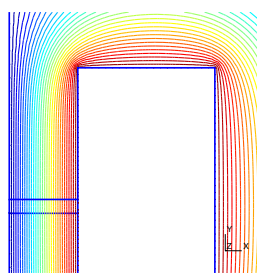

(c) MMF model sol.
Fig. 3. Current source in a slot with air gap (portion of a device): field lines with (a)-(b) classical full model and (c) MMF model.

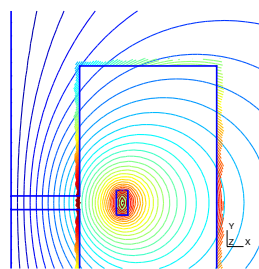

(a) BS SF, FE layer

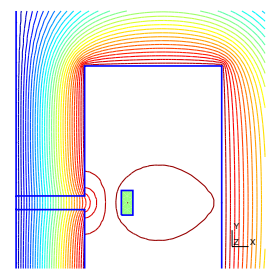

(b) BS-SF model sol.

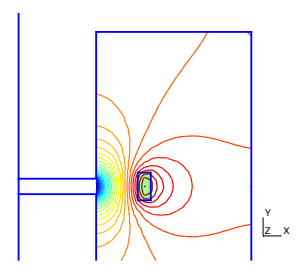

(c) in-slot BS-SF sol.
Fig. 4. Current source in a slot with air gap: field lines with BS-SF model; the BS SF is only needed in an FE layer along core boundary $\Gamma_{m}$; field discontinuities appear (b) through $\Gamma_{m}$ because the total field is obtained in $\Omega_{m}$ whereas the reaction field is obtained outside; due to the simplified shape of the coil, the field distribution in the slot (c) is far from the actual one (Fig. 3b).

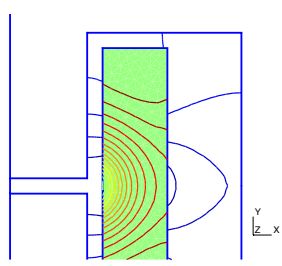

(a) volume correction

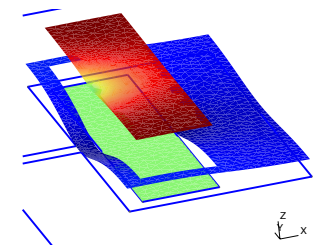

(b) elevation of volume correction
Fig. 5. (a) Field lines of volume correction of coil and its surrounding from BS-SF solution, (b) with its elevation (z-component of a) pointing out the field trace discontinuities; the field distribution (a) is now correct: the total field in the actual coil (same as in Fig. 3b) and the reaction field elsewhere.

\section{ACKNOWLEDGMENT}

This work is supported by the F.R.S.-FNRS and the CNPq (project 400452/2014-6).

\section{REFERENCES}

[1] P. Ferrouillat, C. Guérin, G. Meunier, B. Ramdane, P. Labie, D. Dupuy, "Computation of Source for Non-Meshed Coils with A-V Formulation Using Edge Elements," IEEE Trans. Magn., vol. 51, 2015, to appear.

[2] P. Dular, R. V. Sabariego, M. V. Ferreira da Luz, P. Kuo-Peng L. Krähenbühl, "Perturbation finite element method for magnetic model refinement of air gaps and leakage fluxes," IEEE Trans. Magn., vol. 45, no. 3, pp. 1400-1403, 2009.

[3] P. Dular, L. Krähenbühl, R.V. Sabariego, M. V. Ferreira da Luz, P. KuoPeng and C. Geuzaine. "A finite element subproblem method for position change conductor systems", IEEE Trans. Magn., vol. 48, no. 2, pp. 403-406, 2012 .

[4] P. Dular, V. Péron, R. Perrussel, L. Krähenbühl, C. Geuzaine. "Perfect conductor and impedance boundary condition corrections via a finite element subproblem method," IEEE Trans. Magn., vol. 50, no. 2, paper 7000504, 4 pp., 2014 